\title{
Clinical predictors of malignancy in patients diagnosed with atypical ductal hyperplasia on vacuum-assisted core needle biopsy
}

\author{
Diana Hodorowicz-Zaniewska ${ }^{1}$, Karolina Brzuszkiewicz ${ }^{1}$, Joanna Szpor' ${ }^{2}$, Wojciech Kibil ${ }^{1}$, Andrzej Matyja ${ }^{1}$, \\ Katarzyna Dyląg-Trojanowska ${ }^{3}$, Piotr Richter ${ }^{1}$, Antoni M. Szczepanik ${ }^{1}$ \\ ${ }^{1}$ First Chair of General Surgery, Department of General, Oncological and Gastroenterological Surgery, Jagiellonian University Medical \\ College, Krakow, Poland \\ ${ }^{2}$ Chair of Pathomorphology, Jagiellonian University Medical College, Krakow, Poland \\ ${ }^{3}$ Department of General and Oncological Surgery, Hospital, Sucha Beskidzka, Poland
}

Videosurgery Miniinv 2018; 13 (2): 184-191

DOI: https://doi.org/10.5114/wiitm.2018.73528

\begin{abstract}
Introduction: Atypical ductal hyperplasia $(A D H)$ is a benign lesion, which due to the risk of coexisting cancer is classified as a lesion of uncertain malignant potential.

Aim: To identify clinical predictors of cancer underestimation in patients with ADH diagnosed after vacuum-assisted breast biopsy (VABB).

Material and methods: Between 2001 and 2016, a total of 3804 vacuum-assisted core needle biopsies were performed at the First Chair of General Surgery of the Jagiellonian University Medical College in Krakow, including 2907 ultrasound (US)-guided biopsies and 897 digital stereotactic procedures. Seventy-six women were diagnosed with $A D H$ and 72 of them underwent subsequent surgical excision. Demographic factors, medical history, family history, clinical symptoms, type and size of lesion determined in imaging scans, size of biopsy needle, and presence of coexisting lesions in VABB specimens were analysed as potential predictors of malignancy underestimation.

Results: Underestimation of breast carcinoma occurred in 21 (29.2\%) patients. The upgrade rate was significantly higher only in patients with a lesion visible both in mammography (MMG) and US examinations and combined BIRADS-5.

Conclusions: Vacuum-assisted core needle biopsy is a minimally invasive technique used in diagnosing ADH. As the risk of breast malignancy underestimation is relatively high, open surgical biopsy remains the recommended procedure, especially in patients with lesions detected both in mammography and US examination. As we could not identify the factors that preclude cancer underestimation, all the women diagnosed with $A D H$ should be informed about the risk of cancer underestimation.
\end{abstract}

Key words: atypical ductal hyperplasia, breast cancer underestimation, vacuum-assisted core needle biopsy.

\section{Introduction}

Atypical ductal hyperplasia (ADH) is a benign proliferative lesion, but due to a risk of coexisting cancer it is classified as B3 diagnostic category, covering lesions of uncertain malignant potential [1, 2]. It is characterized by proliferation of monomorphic, evenly distributed epithelial cells in the terminal ductal-lobular unit. Atypical ductal hyperplasia resembles low-grade ductal carcinoma in situ (DCIS), but it dif-

\section{Address for correspondence}

Karolina Brzuszkiewicz MD, First Chair of General Surgery, Department of General, Oncological and Gastroenterological Surgery, Jagiellonian University Medical College, 40 Kopernika St, 31-501 Krakow, Poland, phone: +48 608518 410, e-mail: karola.brzuch@gmail.com 
fers by an admixed population of non-monomorphic cells or by a size limit - involvement of less than two duct spaces or an area of less than $2 \mathrm{~mm}$ in diameter $[3,4]$.

The definition of atypical ductal hyperplasia is based on excisional breast biopsy specimens and due to the limited material obtained from standard core-needle biopsy (CNB), a definitive diagnosis in such cases is hardly possible [3]. The decision to perform surgical excision of the lesion diagnosed as $\mathrm{ADH}$ after CNB is justified by a $30-54 \%$ incidence of DCIS or invasive breast carcinoma in the material from the surgical specimens [2, 5-7]. Vacuum-assisted core needle biopsy (vacuum-assisted breast biopsy - VABB) provides bigger samples, and therefore the underestimation of malignancy is distinctly lower, in the range 9-36\% [8-11].

Factors increasing the risk of cancer underestimation include breast symptoms, findings in mammography other than microcalcifications alone, size of biopsy needle, number of biopsy samples, coexisting intraductal papilloma, diagnosis of marked atypia and experience level of the pathologist [6, 12]. There is also a higher risk of underestimation in patients over 60 years of age, with family history of breast cancer, or in patients who had breast cancer before [9, 13].

Although there are no doubts regarding the recommendations for excisional biopsy, in the case of $A D H$ diagnosed with $C N B$, such indications for VABB are inconsistent [8, 14-18].

As reported in the literature, the chances for underestimation become distinctly lower when multiple samples are taken and the lesion removal on post-biopsy mammograms is complete [8, 14-16].

\section{Aim}

The aim of this study was to identify clinical predictors of cancer underestimation in patients with ADH diagnosed after VABB.

\section{Material and methods}

Between 2001 and 2016, in the Regional Outpatient Clinic of Early Diagnosis and Treatment of Breast Diseases at the First Chair of General Surgery of the Jagiellonian University Medical College in Krakow, a total of 3804 vacuum-assisted core needle biopsies were performed. They included 2907 US-guided biopsies and 897 digital stereotactic procedures. Of these, 76 (1.99\%) women were diagnosed with $\mathrm{ADH}$. The rate of procedure-related complications was about $1.5 \%$, with haematoma being the most common adverse event.

In the whole study population, $\mathrm{ADH}$ was diagnosed in $1.6 \%$ of patients who underwent US-guided biopsy, and in 3.1\% subjected to stereotactic biopsy. The rate of ADH diagnosis in patients in whom only microcalcifications were diagnosed was $6.6 \%$ (28/421). In cases where the lesion was not visible in US, stereotactic digital mammography-guided biopsy was performed. Other patients underwent US-guided biopsies.

Seventy-two patients from the group of $76 \mathrm{pa}-$ tients diagnosed with ADH underwent subsequent surgical excision and were followed up with mammography/US examinations after six months and then annually independently of the final diagnosis. The follow-up ranged from 18 months to 15 years, the average being 6.7 years. Four women who were not subjected to open surgical biopsy were excluded from the analysis. Two of them refused the procedure and were followed up only, one was diagnosed with synchronous breast carcinoma, and the fourth one underwent further treatment in another centre.

All patients underwent breast ultrasound and mammography. Clinical data were obtained from their medical records and included patient's age, menopausal status, family history of breast cancer, history of previous or presence of synchronous breast carcinoma, clinical symptoms (nipple discharge, pain, itching), type and size of lesion determined in mammography and/or in US, Breast Imaging and Reporting Data System (BIRADS) classification of mammographic and US findings, presence of microcalcifications, and size of biopsy needle. Presence of coexisting intraductal papilloma or fibroadenoma in VABB specimens was also recorded.

Core biopsies were performed using 7 G (23 cases $-31.9 \%$ ), and $10 \mathrm{G}$ or $11 \mathrm{G}$ (49 patients $-68.1 \%)$ needles. The number of biopsy specimens ranged from 5 to 14, i.e. 7 on average.

The specimens were fixed in $10 \%$ neutral buffered formalin solution and sent for histopathological evaluation to the Chair of Pathomorphology of the Jagiellonian University Medical College. For the purpose of this study, all biopsy specimens were verified by a second pathologist who was not aware of the post-excisional pathologic results. 
Table I. Clinical and morphological features of the study group

\begin{tabular}{|c|c|c|}
\hline $\begin{array}{l}\text { Clinical and morphological } \\
\text { features of biopsy specimens }\end{array}$ & $\begin{array}{l}\text { Number of } \\
\text { cases }\end{array}$ & $\begin{array}{l}\text { Percentage } \\
\text { of patients }\end{array}$ \\
\hline \multicolumn{3}{|l|}{ Age: } \\
\hline$\leq 60$ & 55 & 76.4 \\
\hline$>60$ & 17 & 23.6 \\
\hline \multicolumn{3}{|l|}{ Menopause: } \\
\hline Yes & 44 & 61.1 \\
\hline No & 28 & 38.9 \\
\hline \multicolumn{3}{|l|}{ Family history of disease: } \\
\hline Yes & 13 & 18.1 \\
\hline No & 59 & 81.9 \\
\hline \multicolumn{3}{|c|}{ History of synchronous/undergone breast cancer: } \\
\hline Yes & 4 & 5.6 \\
\hline No & 68 & 94.4 \\
\hline Asymptomatic lesion & 47 & 65.3 \\
\hline Symptomatic lesion & 25 & 34.7 \\
\hline \multicolumn{3}{|c|}{ Lesion size (the largest size in USG/MMG) [mm]a: } \\
\hline$<10$ & 37 & 51.4 \\
\hline $10-20$ & 20 & 27.8 \\
\hline$>20$ & 12 & 16.7 \\
\hline Lesion visible only in USG & 18 & 25 \\
\hline Lesion visible only in MMG & 23 & 31.9 \\
\hline $\begin{array}{l}\text { Lesion visible in both examina- } \\
\text { tions }\end{array}$ & 31 & 43.1 \\
\hline \multicolumn{3}{|c|}{ Papilloma or fibroadenoma co-diagnosis: } \\
\hline Yes & 18 & 25 \\
\hline No & 54 & 75 \\
\hline
\end{tabular}

USG - ultrasonography, MMG - mammography. aln 3 patients the size of the lesion was not calculated.

Twenty-eight (38.9\%) of the studied 72 biopsies were stereotactic and 44 (61.1\%) US-guided. The mean age of patients in the study group was 53.4 \pm 11.56 years and ranged from 28 to 79 years. The clinical characteristics of the study group are summarized in Table I.

Morphologically, there were 44 (61.1\%) solid masses detected at US examination, while the mammography revealed: 25 (35.8\%) masses, 24 clusters or areas of microcalcifications (35.8\%) and 3 architectural distortions (4.5\%) (Table II).

\section{Statistical analysis}

For each analysed predictive factor, an odds ratio (OR) was calculated with $95 \%$ confidence interval $(95 \% \mathrm{Cl})$. For multifactorial analysis, a binary logit model of logistic regression was applied. The level of significance was set at $p \leq 0.05$. Statistical calculations were performed using the Statistica 10 package.

\section{Results}

For the purpose of this study, all biopsy specimens were assessed by a second pathologist who was blinded to the final diagnosis to confirm the initial finding of ADH.

In 2 cases, the results of the second pathologic examination of the VABB specimens revealed moderate suspicion of DCIS, but not invasive cancer.

In the group of 72 women who underwent surgical excision, the final pathologic report revealed non-malignant lesions in 51 (70.8\%) and 21 (29.2\%) malignant lesions.

The benign findings were ADH in 13 patients, usual ductal hyperplasia (UDH) in 20 patients, 12 intraductal papillomas, 5 fibroadenomas and 2 lobular carcinomas in situ (LCIS). In the group of malignant lesions, there were 11 invasive ductal carcinomas and 10 cases of DCIS. The mean age of women with malignant lesions was $56.8 \pm 13.02$ years and ranged from 34 to 78 years. In the group of benign lesions the mean age of patients was $52 \pm 10.73$ years and ranged from 28 to 75 years. The analysis of BIRADS score obtained from mammography and US showed that because some of the lesions were visible in one of the imaging methods only (16 not visible in MMG and 29 not visible in US), a substantial number of cases should not be included in the analysis. Therefore, the highest BIRADS score in either MMG or US was adopted as the combined BIRADS. In the group of invasive cancers, 6 patients were assessed as BIRADS 5 and in the DCIS group, 2 (Table III). Only 1 patient with a benign lesion received BIRADS 5 in US. None of the patients with malignant lesion was assessed as BIRADS $1-3$, but only 3 patients with a benign lesion were within these BIRADS limits.

Multiple clinical data were analysed as potential predictors of malignancy underestimation (Table IV). Among these factors, the presence of clinical symp- 
Table II. Morphology of ADH in imaging examinations (MMG, USG) in the study group $(N=72)$

\begin{tabular}{|lccc|}
\hline Type of examination & Morphology of lesion & $N$ & $\%$ \\
\hline USG & Solid lump & 43 & 59.7 \\
\cline { 2 - 4 } & No lesion & 23 & 31.9 \\
\cline { 2 - 4 } & Mass & 32 & 44.4 \\
\cline { 2 - 4 } & Mass and cluster/area of microcalcifications & 31 & 43.1 \\
\cline { 2 - 4 } & Cluster/area of microcalcifications & 22 & 30.1 \\
\hline Lesions visible in both USG and MMG & Architectural distortion & 4 & 25 \\
\hline
\end{tabular}

ADH - atypical ductal hyperplasia, MMG - mammography, USG - ultrasonography.

Table III. Clinical and pathological features of breast lesions in the patients diagnosed with invasive carcinoma

\begin{tabular}{|c|c|c|c|c|c|}
\hline No. & MMG & USG & $\begin{array}{l}\text { Max. size } \\
{[\mathrm{mm}]}\end{array}$ & BIRADS & Core needle biopsy \\
\hline 1 & Microcalcifications + tumour & Tumour & 40 & 5 & Marked atypia \\
\hline 2 & Tumour & Tumour & 26 & 5 & Marked atypia \\
\hline 3 & Tumour & Tumour & 12 & $4 a$ & Moderate atypia \\
\hline 4 & Microcalcifications + tumour & Tumour & 15 & 5 & Marked atypia \\
\hline 5 & Microcalcifications + tumour & Tumour & 40 & 5 & Marked atypia \\
\hline 6 & Microcalcifications + tumour & Tumour & 13 & 5 & Moderate atypia \\
\hline 7 & No changes & Tumour & 11 & $4 b$ & Moderate atypia \\
\hline 8 & Tumour & Tumour & 35 & 5 & Marked atypia \\
\hline 9 & Tumour & Tumour & 8 & $4 c$ & Marked atypia \\
\hline 10 & Microcalcifications + tumour & Tumour & 7 & $4 c$ & $\begin{array}{c}\text { Marked atypia } \\
\text { Coexisting intraductal papilloma }\end{array}$ \\
\hline 11 & Tumour & Tumour & 5 & $4 a$ & Marked atypia \\
\hline
\end{tabular}

$M M G$ - mammography, USG - ultrasonography.

toms $(O R=2.9)$, the visibility of the lesion in both imaging methods $(O R=4.37)$, the visibility of the lesion in MMG $(O R=10.9)$, the size over $1 \mathrm{~cm}(O R=$ 3.41) and the combined BIRADS -5 (OR = 15.62) were assessed as statistically significant predictive factors. Neither age nor menopausal status was a predictor of malignancy underestimation. Patient's history and family history had no impact on cancer underestimation either. Interestingly, no differences in diagnostic accuracy were found for the size of the biopsy needles.

The multifactorial regression model was used to evaluate the predictive factors mentioned above. Only the visibility of the lesion in both diagnostic methods $(p=0.04)$ and combined BIRADS-5 $(p=0.02)$ were independent predictors in this model.

\section{Discussion}

Atypical ductal hyperplasia is a benign proliferative breast disease, but due to the risk of coexisting cancer it is classified as a lesion of uncertain malignant potential. In women diagnosed with $\mathrm{ADH}$, the risk of breast cancer is 3-5-times higher than in the general population $[19,20]$. A positive family history in association with ADH diagnosis has been shown to increase this risk even 10 times above baseline [21]. Therefore, women diagnosed with ADH are subjected to follow-up. The diagnosis of $A D H$ is mainly the consequence of breast cancer screening, and in the minority of patients it results from diagnostic procedures for breast-related symptoms or a lump. 
Diana Hodorowicz-Zaniewska, Karolina Brzuszkiewicz, Joanna Szpor, Wojciech Kibil, Andrzej Matyja, Katarzyna Dyląg-Trojanowska, Piotr Richter, Antoni M. Szczepanik

Table IV. Factors affecting underestimation of diagnosis of breast cancer

\begin{tabular}{|lcccc|}
\hline Analysed parameter & Odds ratio & $95 \%$ confidence interval & $Z$ statistic & $P$-value \\
\hline Age $>$ 60 years & 2.8718 & $0.9207-8.9575$ & 1.818 & 0.0691 \\
\hline Menopause & 1.4 & $0.4826-4.0611$ & 0.619 & 0.5358 \\
\hline Clinical symptoms (nipple discharge, pain, itching) & 2.9071 & $1.0129-8.3437$ & 1.984 & 0.0473 \\
\hline Needle size 10 (11) G vs. 7 G & 1.2500 & $0.4113-3.7985$ & 0.394 & 0.6939 \\
\hline Lesion visible in USG & 0.9459 & $0.3059-2.9253$ & 0.096 & 0.9231 \\
\hline Lesion visible in MMG and USG & 4.3750 & $1.4812-12.9227$ & 2.671 & 0.0076 \\
\hline Lesion visible in MMG (including microcalcifications) & 10.9091 & $1.3508-88.1023$ & 2.242 & 0.0250 \\
\hline Coexisting intraductal papilloma or fibroadenoma & 0.2303 & $0.0478-1.1096$ & 1.830 & 0.0672 \\
\hline Coexisting intraductal papilloma & 0.1818 & $0.0219-1.5092$ & 1.579 & 0.1144 \\
\hline Family history of breast cancer & 0.7468 & $0.2696-2.0681$ & 0.562 & 0.5742 \\
\hline History of breast cancer & 8.3333 & $0.8137-85.3463$ & 1.786 & 0.0741 \\
\hline Lesion size over 1 cm & 3.4125 & $1.0081-11.5515$ & 1.973 & 0.0485 \\
\hline BIRADS 5 & 15.62 & $1.6976-143.8144$ & 2.427 & 0.0152 \\
\hline
\end{tabular}

$M M G$ - mammography, USG - ultrasonography.

In our series of patients (data not shown), $34.7 \%$ of patients were symptomatic, whereas $20.8 \%$ had palpable lesions. A focal finding in one of the imaging studies is an indication for biopsy.

Atypical ductal hyperplasia is most commonly diagnosed based on biopsy results of microcalcifications detected in the screening mammography. Atypical ductal hyperplasia does not have typical morphological features in ultrasonography and it is detected in US-guided biopsy in about $1 \%$ of cases [22]. In breast MR, which should be used as a diagnostic adjunct to US and MMG in women with greater lifetime risk of developing breast carcinoma [23], it can manifest as a small segmental or ductal focal enhancement [24].

Atypical ductal hyperplasia is found in $3-10 \%$ of the CNB performed for microcalcifications [7, 13, $25]$. In the analysed group of patients, ADH was diagnosed in $3.1 \%$ of all stereotactic procedures, and in $6.6 \%$ of biopsies performed for microcalcifications, which is in line with the findings reported in the literature. The incidence of ADH in US-guided biopsies was $1.6 \%$.

For years, open surgical biopsy has been the best option of further treatment for $\mathrm{ADH}$. It is however an invasive procedure associated with excision of breast tissue and it may cause breast deformation and scarring. About $70 \%$ of women will probably not benefit from surgical excision, as it does not reduce the risk of cancer in the same or contralateral breast $[18,26]$. Moreover, the finding of ADH may affect the risk of breast cancer development to a lesser degree than previously reported [27].

Minimally invasive techniques, including the vacuum-assisted core needle biopsy, are an interesting diagnostic and therapeutic alternative. The VABB turned out to be a low-invasive technique, well tolerated by patients, effective, and with a low rate of complications. Due to the possibility of obtaining larger tissue sections, the risk of complications in the case of VABB is slightly higher than in the case of core biopsy, mainly the risk of haematoma [28]. However, among 3804 patients who underwent $\mathrm{VABB}$, only 3 patients required surgical treatment of haematoma. Among other complications were fat necrosis and Mondor's disease [29]. Therefore, the advantage of acquiring larger samples significantly outweighs the risks associated with VABB.

Diagnostic accuracy of VABB ranges between $98 \%$ and $100 \%$ for solid lesions [30]. At the same time, it is less invasive and cost-effective compared to surgical excision.

The usefulness of minimally invasive biopsy techniques in diagnosis and treatment of ADH has been a subject of analyses for a long time, but the main problem is the relatively high risk of breast cancer 
underestimation. The simple strategy of its management consists of reduction of indications for unnecessary surgery without compromising early diagnosis of malignancy. Rates of false-negative diagnoses of breast carcinoma in cases yielding $A D H$ in $C N B$ reported in the literature reach $9-54 \%$ [5-11, 13]. The difference between core needle biopsy and vacuum-assisted biopsy is related to the volume of the biopsy specimens and to the biopsy needle diameter [31]. For VABB performed with 11-7G size needles, the underestimation risk seems slightly lower than for CNB and is $9-36 \%[8-11,13]$.

The results obtained from our group of patients are consistent with the findings of other authors. A comparison of histopathological results of VABB and surgical excision demonstrated $29.2 \%$ underestimation, almost equally divided between DCIS and invasive ductal carcinoma. What is interesting, the use of $7 \mathrm{G}$ needles did not provide better results than for $10 \mathrm{G}$ or $11 \mathrm{G}$ needles. Similar observations were reported by Eby et al., with respect to the needle diameters of $9 \mathrm{G}$ and $11 \mathrm{G}$ [32].

The suggested inferiority of CNB due to the volume of obtained biopsy caused by the smaller diameter of the needle plays a role only in the comparison of CNB and VABB, while the size of the biopsy needle in $V A B B$ does not affect the underestimation risk.

In the recent years, there have been a number of interesting studies suggesting that in the selected groups of patients with the ADH diagnosed after a $V A B B$, the avoidance of surgical excision is justified [8, 14-18].

Forgeard et al. identified three subsets of patients [14]. The first consisted of those with $\mathrm{ADH}$ in microcalcifications on an area of less than $6 \mathrm{~mm}$ in diameter, showing complete removal in post-biopsy mammograms. In this group of patients, no case of underestimation was reported. The second group, with low risk of coexisting malignancy (below 4\%), included cases with fewer than 2 foci of $\mathrm{ADH}$ in microcalcifications on an area of 6-21 mm or diameter of less than $6 \mathrm{~mm}$, but showing incomplete macroscopic removal. The third group, with the risk of breast cancer underestimation between $36 \%$ and $38 \%$, consisted of women with a diagnosis of more than two foci of atypical hyperplasia, the removal of which was not radical, and cases of ADH in microcalcifications on an area with a diameter of more than $21 \mathrm{~mm}$. For the first two groups, Forgeard suggested a follow-up program without surgical ex- cision. In group 3, an open surgical biopsy remained a standard [14].

A similar subset of patients was analysed by Caplain et al. [17] In 124 patients with diagnosed $\mathrm{ADH}$, the underestimation of breast cancer of $28 \%$ was reported. Women with microcalcifications below $6 \mathrm{~mm}$ showing complete removal in radiological examination were followed up. While alternative treatment might be applied in the case of lesions on an area of $6-21 \mathrm{~mm}$, or with at least 2 foci of $A D H$, for the lesions above $21 \mathrm{~mm}$, surgery should be an absolute recommendation.

Nguyen et al. pointed to the role of clinical and pathological correlation, and included in the follow-up only patients with ADH diagnosed based on biopsies of isolated and completely removed (> 95\%) microcalcifications without features of marked atypia and/or necrosis in histology [15]. All cases of solid lesions coexisting with microcalcifications were in their opinion at high risk of underestimation, and surgical excision was mandatory. Their observations are similar to our findings. In our study, the presence of the lesion in both US and MMG was an independent predictive factor for malignancy.

The other independent predictor of malignancy in our study was the classification as BIRADS 5 in either US or MMG. This is in concordance with some authors [33], but others did not confirm this finding [34]. In our study, the combined BIRADS has been used e.g. for the higher score of US and MMG, which is different from the score used by others. Therefore, our findings are not fully comparable with the cited studies.

As shown in our study, the size of the findings significantly affected the final histopathological result, and a diameter greater than $1 \mathrm{~cm}$ in a univariate analysis was associated with higher risk of underestimation. Similar results were reported by Chae et al. [20]. In other studies, which identified different subsets of lesions (size $<6 \mathrm{~mm}, 6-21 \mathrm{~mm}$ and $>21 \mathrm{~mm}$ or size $<7 \mathrm{~mm}$ and $>7 \mathrm{~mm}$ ), the risk of $\mathrm{ADH}$ upgrade was substantially higher for findings above 6-7 $\mathrm{mm}[14,17,35]$.

The risk of false-negative diagnoses was related to the age of the patients. However, we did not observe such a phenomenon. This is contrary to the studies by Winchester et al. and Andrales et al., who reported a correlation of age and cancer underestimation in women diagnosed with ADH in biopsy [9, 13]. No correlation with patients' age was found in 
studies of Deshaies et al., Sohn et al. or Graesslin et al. $[6,31,36]$ which suggests that age cannot define a subset of patients who do not require surgical excision.

Andrales et al., as well as other authors, reported that the risk of underestimation correlated with history of previous or synchronous breast carcinoma [9]. However, due to different sizes of analysed groups, it is difficult to obtain conclusive results.

What is interesting in our study is the relatively high percentage of ADH diagnoses in US-guided biopsies (1.6\%). It should be emphasised that the highest risk of underestimation was found for lesions visible in both MMG and US, which was an independent predictive factor $(p=0.038)$. These results indicate that the clinical picture, radiological findings and pathological results should be completely consistent ('the rule of triple assessment'). If histopathologically confirmed ADH is visible in both imaging examinations, it is always necessary to insist on surgical excision.

Atypical ductal hyperplasia is a rare diagnosis in CNB or VABB; therefore the majority of studies are based on groups including 50-150 patients. Some of these studies present a model of prediction which can indicate the group of patients in whom the follow-up without surgical excision is safe [37, 38]. However, one should consider the differences between groups of patients and, what is more important, quite high inter-observer variability in pathologic diagnosis of ADH. Current guidelines cannot precisely identify such factors that justify only observation of ADH patients [39]. Our study also has such limitations. Even the studies based on some additional molecular parameters did not reduce the underestimation rate significantly [40].

\section{Conclusions}

The vacuum-assisted core needle biopsy is one of the minimally invasive techniques used in diagnosing $\mathrm{ADH}$. As the risk of breast malignancy underestimation is relatively high (29.2\%), open surgical biopsy remains the recommended procedure, especially in patients with lesions detected both in mammography and US examination. As we could not identify the factors that preclude cancer underestimation, all the women who have the diagnosis of ADH in VABB should be informed about the risk of cancer underestimation.

\section{Conflict of interest}

The authors declare no conflict of interest.

\section{References}

1. Chmielik E, Łuczynska E. [Core needle biopsy-diagnostic guidelines]. Pol J Pathol 2009; 3: 13-9.

2. Ellis IO, Humphreys S, Michell M, et al. Guidelines for breast needle core biopsy handling and reporting in breast screening assessment. J Clin Pathol 2004; 57: 897-902.

3. Tavassoli FA, Norris HJ. A comparison of the results of longterm follow-up for atypical intraductal hyperplasia and intraductal hyperplasia of the breast. Cancer 1990; 65: 518-29.

4. Schnitt SJ, Ellis IO, van de Vijver MJ, et al. Intraductal proliferative lesions: introduction and overview. In: WHO Classification of Tumours of the Breast. Lakhani SR, Ellis IO, Schnitt SJ, Tan PH, van de Vijver MJ (eds.). IARC Press, Lyon 2012; 82-94.

5. Rosen PP, Hoda SA. Breast pathology. In: Breast Pathology: Diagnosis by Needle Core Biopsy. Wolters Kluwer Health/ Lippincott Wiliams \&Wilkins. Philadelphia 2010; 171-86.

6. Deshaies I, Provencher L, Jacob S, et al. Factors associated with upgrading to malignancy at surgery of atypical ductal hyperplasia diagnosed on core biopsy. Breast 2011; 20: 50-5.

7. Maganini RO, Klem DA, Huston BJ, et al. Upgrade rate of core biopsy-determined atypical ductal hyperplasia by open excisional biopsy. Am J Surg. 2001; 182: 355-8.

8. Dominici L, Liao GS, Brock J, et al. Large needle core biopsy of atypical ductal hyperplasia: results of surgical excision. Breast J 2012; 18: 506-8.

9. Andrales G, Tirk P, Wallace T, et al. Is surgical excision necessary for atypical ductal hyperplasia of the breast diagnosed by mammotome? Am J Surg 2000; 180: 313-5.

10. Palom K, Murawa D, Kurzawa P, et al. Underestimation of cancer in case of diagnosis of atypical ductal hyperplasia (ADH) by vacuum assisted core needle biopsy. Rep Pract Oncol Radioth 2012; 17: 129-33.

11. Allison KH, Eby PR, Kohr J, et al. Atypical ductal hyperplasia on vacuum-assisted breast biopsy: suspicion for ductal carcinoma in situ can stratify patients at high risk for upgrade. Human Path 2011; 42: 41-50.

12. Doren E, Hulvat M, Norton J, et al. Predicting cancer on excision of atypical ductal hyperplasia. Am J Surg 2008; 195: 358-62.

13. Winchester D, Bernstein JR, Jeske JM, et al. Upstaging of atypical ductal hyperplasia after vacuum-assisted 11-gauge stereotactic core needle biopsy. Arch Surg 2003; 138: 619-23.

14. Forgeard C, Benchaib M, Guerin N, et al. Is surgical biopsy mandatory in case of atypical ductal hyperplasia on 11-gauge core needle biopsy? A retrospective study of 300 patients. Am J Surg 2008; 196: 339-45.

15. Nguyen C, Albarracin CT, Whitman GJ, et al. Atypical ductal hyperplasia in directional vacuum-assisted biopsy of breast microcalcifications: considerations for surgical excision. Ann Surg Oncol 2011; 18: 752-61.

16. McGhan LJ, Pockaj BA, Wasif N, et al. Atypical ductal hyperplasia on core biopsy: an automatic trigger for excisional biopsy? Ann Surg Oncol 2012; 19: 3264-9. 
17. Caplain A, Drouet $\mathrm{Y}$, Peyron M, et al. Management of patients diagnosed with atypical ductal hyperplasia by vacuum-assisted core biopsy: a prospective assessment of the guidelines used at our institution. Am J Surg 2014; 208: 260-7.

18. Jaffer S, Nagi C, Bleiweiss IJ. Should incidental microscopic radiologically occult atypical duct hyperplasia of the breast be excised? Breast J 2014; 20: 103-4.

19. Worsham MJ, Abrams J, Raju U, et al. Breast cancer incidence in a cohort of women with benign breast disease from a multiethnic, primary health care population. Breast J 2007; 13: 115-21.

20. Chae BJ, Lee A, Song BJ, Jung SS. Predictive factors for breast cancer in patients diagnosed atypical ductal hyperplasia at core needle biopsy. World J Surg Oncol 2009; 23: 77-82.

21. Dupont WD, Parl FF, Hartmann WH, et al. Breast cancer risk associated with proliferative breast disease and atypical hyperplasia. Cancer 1993; 71: 1258-65.

22. Coopey SB, Mazzola E, Buckley JM, et al. The role of chemoprevention in modifying the risk of breast cancer in women with atypical breast lesions. Breast Cancer Res Treat 2012; 136: 627-33.

23. Popiela TJ, Kibil W, Herman-Sucharska I, et al. The use of magnetic resonance mammography in women at increased risk for developing breast cancer. Videosurgery Miniinv 2013; 8: 55-62.

24. Molleran VM, Mahoney MC. High-risk Findings in Breast MRI. Elsevier Saunder, Philadelphia 2014; 65.

25. Harvey JM, Sterret F, Frost FA. Atypical ductal hyperplasia and atypia of uncertain significance in core biopsies from mammographically detected lesions: correlation with excision diagnosis. Pathology 2002; 34: 410-6.

26. Polat AK, Kanbour-Shakir A, Andacoglu O, et al. Atypical ductal hyperplasia on core biopsy: is further surgery needed? Am J Med 2012; 344: 28-31.

27. Menes TS, Kerlikowske K, Lange J, et al. Subsequent breast cancer risk following diagnosis of atypical ductal hyperplasia on needle biopsy. JAMA Oncol 2017; 3: 36-41.

28. Hertl K, Marolt-Music M, Kocijancic I, et al. Haematomas after percutaneus vacuum-assisted breast biopsy. Ultraschall Med 2009; 30: 33-6.

29. Kibil W, Hodorowicz-Zaniewska D, Kulig J. Mondor's disease in a patient after a mammotome biopsy. Videosurgery Miniinv 2015; 10: 138-40.

30. Nakano S, Sakamoto H, Ohtsuka M, et al. Evaluation and indications of ultrasound-guided vacuum-assisted core needle breast biopsy. Breast Cancer 2007; 14: 292-6.

31. Sohn V, Arthurs Z, Herbert G, et al. Atypical ductal hyperplasia: improved accuracy with 11-gauge vacuum assisted versus the 14-gauge core biopsy needle. Ann Surg Oncol 2007; 14 2497-501.

32. Eby PR, Ochsner JE, DeMartini WB, et al. Frequency and upgrade rates of atypical ductal hyperplasia diagnosed at stereotactic vacuum-assisted breast biopsy: 9-versus 11-gauge. Am J Roentgenol 2009; 192: 229-34.

33. Wiratkapun C, Wibulpholprasert B, Lertsithichai P, et al. Breast cancer underestimation rate of atypical ductal hyperplasia diagnosed by core-needle biopsy under imaging guidance. J Med Assoc Thai 2005; 88: 460-6.
34. Menes TS, Rosenberg R, Balch S, et al. Upgrade of high-risk breast lesions detected on mammography in the Breast Cancer Surveillance Consortium. Am J Surg 2014; 207: 24-31.

35. Renshaw AA, Cartagena N, Schenkman RH, et al. Atypical ductal hyperplasia in breast core needle biopsies. Am J Clin Pathol 2001; 116: 92-6.

36. Graesslin O, Antoine M, Chopier J, et al. Histology after lumpectomy in women with epithelial atypia in stereotactic vaccumm-assisted breast biopsy. Eur J Surg Oncol 2010; 36: 170-5.

37. Khoury T, Chen X, Wang D, et al. Nomogram to predict the likelihood of upgrade of atypical ductal hyperplasia diagnosed on a core needle biopsy in mammographically detected lesions. Histopathology 2015; 67: 106-20.

38. Ko E, Han W, Lee JW, et al. Scoring system for predicting malignancy in patients diagnosed with atypical ductal hyperplasia at ultrasound-guided core needle biopsy. Breast Cancer Res Treat 2008; 112: 189-95.

39. Rageth CJ, O'Flynn EA, Comstock C, et al. First International Consensus Conference on lesions of uncertain malignant potential in the breast (B3 lesions). Breast Cancer Res Treat 2016; 159: 203-13.

40. Polat AK, Soran A, Kanbour-Shakir A, et al. The role of molecular biomarkers for predicting adjacent breast cancer of atypical ductal hyperplasia diagnosed on core biopsy. Cancer Biomark 2016; 17: 293-300.

Received: 25.09.2017, accepted: 13.11.2017 